\title{
New-Onset Complete Heart Block Later Complicated by Decompensated Heart Failure Following COVID-19 Infection
}

\author{
Katrina Ngo, OMS-III ${ }^{1}$, Sheldon Myers, OMS-III ${ }^{1}$, Ramanna Merla, $\mathrm{MD}^{2}$, Ronald Chang, $\mathrm{MD}^{2}$ \\ ${ }^{1}$ Western University of Health Sciences - COMP, Pomona, CA \\ ${ }^{2}$ Pomona Valley Hospital Medical Center, Pomona, CA
}

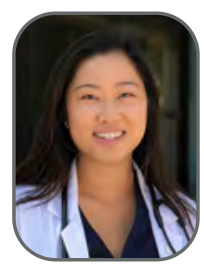

Katrina Ngo, OMS-III

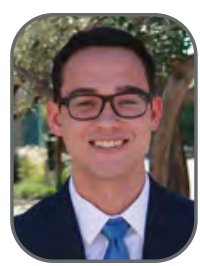

Sheldon Myers, OMS-III

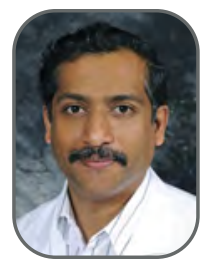

Ramanna Merla, MD

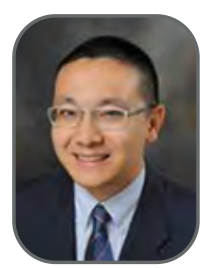

Ronald Chang, MD

\section{CORRESPONDENCE:}

Ronald Chang, MD

Ronald.Chang@pvhmc.org

DOI: $10.38206 / 140109$

\section{KEYWORDS:}

Complete Heart Block, COVID-19, Dilated Cardiomyopathy, Heart Failure with Reduced Ejection Fraction, Myocarditis

\section{ABSTRACT}

Coronavirus disease 2019 (COVID-19) is caused by a strain of coronavirus known as severe acute respiratory syndrome coronavirus $2 .{ }^{1}$ Although respiratory illness remains the main concern regarding COVID-19 infection, multiple other organ systems have been reported to have also been affected, more prominently, the cardiovascular system. ${ }^{3}$ We present a 54-year-old male with a history of newly diagnosed diabetes mellitus type II and chronic kidney disease stage II who presented to the emergency department with progressive dyspnea, myalgias, fever and malaise and was subsequently found to have complete heart block.

\section{INTRODUCTION}

COVID-19 launched a global pandemic which further led to a series of other questions, concerns, and increasing anxiety. There have been over 131 million cases and over 2.8 million deaths worldwide as of April 2021.2 This disease has been confirmed on every continent and has overwhelmed healthcare systems worldwide, peaking at more than 17,000 confirmed COVID-19 admissions in a single day in the United States. ${ }^{8}$ Patients with the symptomatic disease commonly present with cough, myalgias, and headache, with diarrhea and loss of taste and smell also is well documented. ${ }^{7}$ The spectrum of disease is vast and affects many organ systems. Presentation of serious COVID-19 infection is commonly pneumonia (PNA) with fever, dyspnea, and cough leading to acute respiratory distress syndrome. The primary objective in the management of COVID-19 is controlling the systemic inflammation and pulmonary complications of infection. However, case studies have demonstrated sequelae in other organ systems, specifically the cardiovascular system. 3,4 In this report, we describe a patient with presumed normal cardiac structure who was admitted to the hospital with acute COVID-19 PNA and diagnosed with new-onset complete heart block and subsequent decompensated heart failure.

\section{CASE}

Our patient is a 54-year-old male with a history of newly diagnosed diabetes mellitus type 2 and chronic kidney disease (CKD) stage 2 presented to the Emergency Department (ED) with progressive dyspnea, myalgias, fever, and malaise. He was admitted for COVID-19 acute hypoxic respiratory failure and acute kidney injury on 
CKD. The EKG showed complete heart block. (Figure 1) After acute onset bradycardia, a repeat EKG revealed that the interventricular conduction delay had progressed to a new-onset complete heart block. The patient was started on $5 \mathrm{~L}$ nasal cannula for acute hypoxic respiratory failure and was seen by cardiology the following day. No acute interventions were recommended at the time of hospitalization; general COVID-19 management with steroid support, anticoagulation, ribavirin, KALETRA ${ }^{\circledR}$, and INF was continued, and heart block was evaluated for resolution. During electrophysiology consultation the same day, the patient was evaluated at the bedside and was able to ambulate without difficulty breathing or significant changes in heart rate. He was informed that he would need a pacemaker if the heart block did not resolve independently. A dual chamber pacemaker was implanted one week later.

\section{FIGURE 1.}

EKG Showing Complete Heart Block.

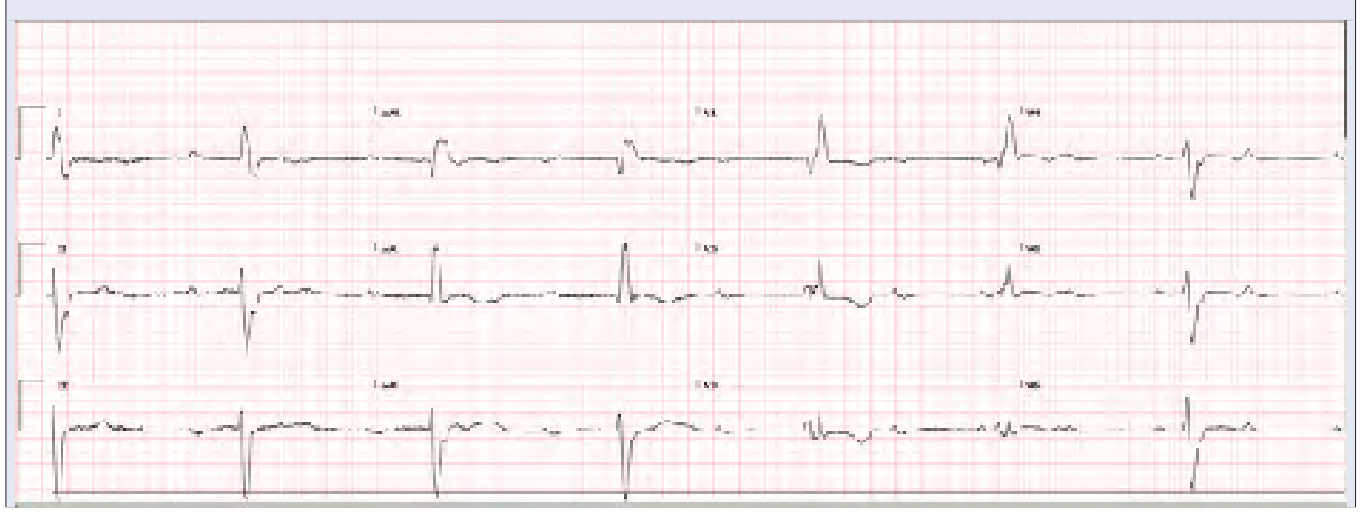

\section{FIGURE 2.}

Repeat EKG Following Dual Chamber Pacemaker.

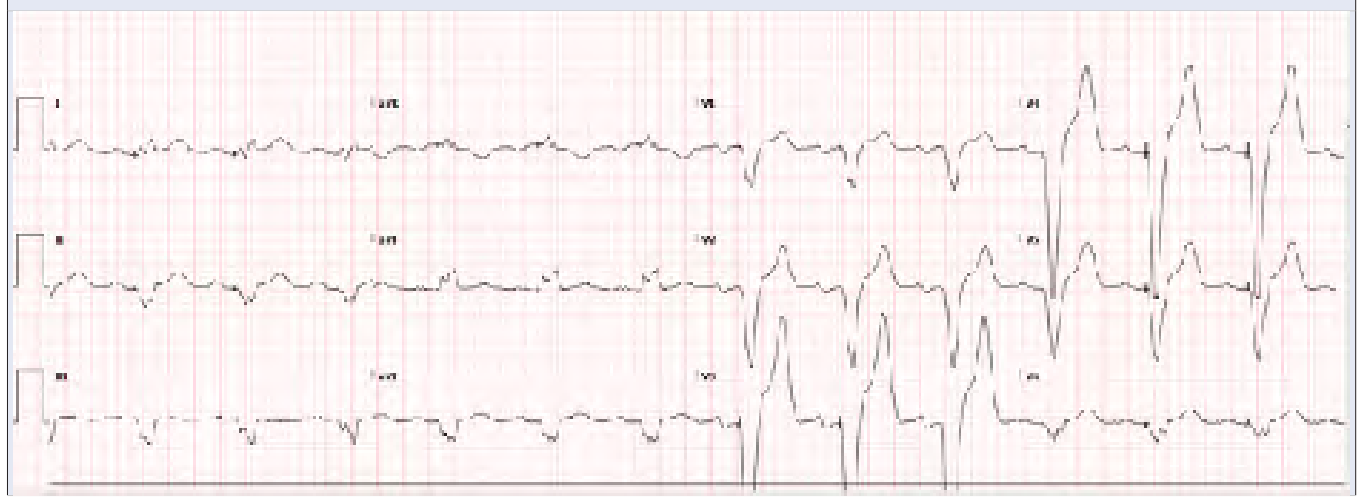

\section{Following placement, repeat EKG}

revealed atrial sensed and ventricular paced rhythm. (Figure 2) Kidney function and $\mathrm{O} 2$ saturation were improved by the end of his hospital stay, and the patient was discharged home to follow up outpatient. (Table 1)

Just under two months after discharge, the patient was readmitted and diagnosed with acute on chronic decompensated heart failure, NY Heart Association class III stage C. An echocardiogram upon admission revealed an ejection fraction $<20 \%$, severely decreased left ventricular systolic function, dilated cardiomyopathy with global hypokinesis, and restrictive left ventricular filling. In addition, the patient continued to be in complete heart block and pacing throughout this second visit. The patient was stabilized and discharged on Carvedilol $3.125 \mathrm{mg} 1 \mathrm{tab}$ PO BID, Furosemide $40 \mathrm{mg} 1$ tab PO BID, and Sacubitrilvalsartan 24mg/26mg 1 tab, PO BID.

\section{TABLE 1.}

Lab Values upon Initial Admission (7/20) and Readmission (9/22)

\begin{tabular}{|l|c|c|c|c|c|}
\hline & $\mathbf{7 / 2 0}$ & $\mathbf{7 / 2 1}$ & $\mathbf{7 / 2 8}$ & $\mathbf{9 / 2 1}$ & $\mathbf{9 / 2 4}$ \\
\hline $\begin{array}{l}\text { SARS-CoV-2 Xpert } \\
\text { Xpress }\end{array}$ & Detected & & & & \\
\hline Glucose & 96 & 259 & & & 112 \\
\hline BUN & 29 & 46 & & & 32 \\
\hline Cr & 2.5 & 2.8 & & & 1.6 \\
\hline eGFR (non-AA) & 28 & 24 & & & 47 \\
\hline Hgb A1c & 7.1 & & & & \\
\hline D-dimer & & & 334 & 542 & 508 \\
\hline Procalcitonin & 4.31 & 4.95 & $<0.05$ & & \\
\hline Troponin-I & $0.23-0.3$ & 0.2 & $<0.03$ & 0.37 & 0.29 \\
\hline BNP & 100 & 87 & 105 & 2013 & 1107 \\
\hline
\end{tabular}




\section{CONCLUSION}

We describe a case of a 54-year-old male diagnosed with COVID-19 PNA and a new-onset complete heart block later complicated by decompensated heart failure. Heart block etiology is widely varied. Physiologically enhanced vagal tone can lead to slow sinus rates and potential development of aberrations in AV conductance. Pathophysiologic heart block can be idiopathic or associated with cardiac disease. Myocarditis-associated heart block is commonly seen with various infectious conditions, including Lyme disease, Rheumatic fever, bacterial endocarditis, and more recently, COVID-19.

The COVID-19 induced heart block mechanism is not well elucidated; however, it is suspected that it may be due to edema and damage to the conduction tissue from direct viral damage, immune-mediated inflammation, ${ }^{5}$ or from hypoxemia and electrolyte abnormalities. ${ }^{3}$ Heart block from COVID-19 infection has been well documented in other case reports. ${ }^{3,4}$ In those reports, the heart block improved with the resolution of their COVID-19 infection. The authors purported that the patients might not have long-term effects of the heart block, considering no pacemaker placement was needed. This is contrary to our patient, who required pacemaker placement for heart block resolution after his COVID-19 infection resolved and his complete heart block persisted. In addition, our patient was asymptomatic, showing no signs of heart block such as syncope and dizziness. Furthermore, our patient returned to the hospital two months later in acute on chronic decompensated heart failure, demonstrating the potential lasting effects of the heart block due to myocarditis.

Among the various sequelae of infection, COVID-19 has been documented to cause exacerbation of existing heart failure. ${ }^{6}$ Our patient had no documented past medical history of any heart conditions before he presented to the ED with COVID-19 PNA. Whether resolving COVID-19 induced cardiac damage after pacemaker placement led to a new-onset heart failure or exacerbated underlying heart failure is unclear.

\section{AUTHOR DISCLOSURES:}

No relevant financial affiliations or conflicts of interest.

\section{REFERENCES:}

1. Nishiga, M., Wang, D.W., Han, Y. et al. COVID-19 and cardiovascular disease: from basic mechanisms to clinical perspectives. Nat Rev Cardiol 17, 543-558 (2020). https://doi.org/10.1038/s41569-020-0413-9

2. WHO coronavirus (COVID-19) Dashboard. https:// covid19. who.int/?gclid=CjOKCQjwmcWDBhCOA RlsALgJ2Qcm6O8oeWBYBX7xkUA1QMSbZhkz vuK7WS8oxC-OpjQ5uYGqjahRsYoaApZpEALw_ wcB. Accessed April 11, 2021.

3. Kochav SM, Coromilas E, Nalbandian A, Ranard LS, Gupta A, Chung MK, Gopinathannair R, Biviano AB, Garan H, Wan EY. Cardiac Arrhythmias in COVID-19 Infection. Circ Arrhythm Electrophysiol. 2020 Jun;13(6):e008719. doi: 10.1161/CIRCEP.120.008719. Epub 2020 May 20. PMID: 32434385; PMCID: PMC7299099.

4. Iqbal El-Assaad, M. Indriati Hood-Pishchany, John Kheir, Kshitij Mistry, Avika Dixit, Olha Halyabar, Douglas Y. Mah, Colin Meyer-Macaulay, Henry Cheng (2020). Complete Heart Block, Severe Ventricular Dysfunction, and Myocardial Inflammation in a Child With COVID-19 Infection. JACC: Case Reports, 2(9), 1351-1355. doi:10.1016/j.jaccas.2020.05.023.

5. Babapoor-Farrokhran S, Gill D, Walker J, Rasekhi RT, Bozorgnia B, Amanullah A. Myocardial injury and COVID-19: Possible mechanisms. Life Sci. 2020;253:117723. doi:10.1016/j.Ifs.2020.117723

6. Fried JA, Ramasubbu K, Bhatt R, et al. The Variety of Cardiovascular Presentations of COVID-19. Circulation. 2020;141(23):1930-1936. doi:10.1161/CIRCULATIONAHA.120.047164

7. Stokes EK, Zambrano LD, Anderson KN, Marder EP, Raz KM, El Burai Felix S, Tie Y, Fullerton KE. Coronavirus Disease 2019 Case Surveillance - United States, January 22-May 30, 2020. MMWR Morb Mortal Wkly Rep. 2020 Jun 19;69(24):759-765. doi: 10.15585/mmwr. mm6924e2. PMID: 32555134; PMCID: PMC7302472.

8. CDC COVID Data Tracker. Centers for Disease Control and Prevention. https://covid.cdc.gov/covid-datatracker/\#new-hospital-admissions. Accessed April 11, 2021. 